\title{
Wavelet Co-movement Significance Testing with Respect to Gaussian White Noise Background
}

\author{
Jitka Poměnková ${ }^{1, *}$, Eva Klejmová ${ }^{1}$, and Tobiáš Malach ${ }^{1}$ \\ ${ }^{1}$ Brno University of Technology, Faculty of Electrical Engineering and Communication, Technická 12, 61600 Brno, Czech Republic
}

\begin{abstract}
The paper deals with significance testing of time series co-movement measured via wavelet analysis, namely via the wavelet cross-spectra. This technique is very popular for its better time resolution compare to other techniques. Such approach put in evidence the existence of both long-run and short-run co-movement. In order to have better predictive power it is suitable to support and validate obtained results via some testing approach. We investigate the test of wavelet power cross-spectrum with respect to the Gaussian white noise background with the use of the Bessel function. Our experiment is performed on real data, i.e. seasonally adjusted quarterly data of gross domestic product of the United Kingdom, Korea and G7 countries. To validate the test results we perform Monte Carlo simulation. We describe the advantages and disadvantages of both approaches and formulate recommendations for its using.
\end{abstract}

\section{Introduction}

Measuring the mutual dependence and co-movement between two inputs (time series or signals) is at the forefront of the interest of many scientific areas such as economy, sociology, biology, geoscience, climatology, engineering, etc. The selection of appropriate analytical instrument comes out from the application area which predefines analytical requirements. In the last decade it became more common an implementation of methods derived from the technical or nature sciences into the social sciences. Therefore, the need of an examination of the possibilities, of an adaptation and the utilization of such a method, the ways of its application and the correct interpretation of the obtained results arise.

The wide range of the methodology implementation can be found in the economics in analysis of globalisation between economies, emerging and financial markets, regional similarities, analysis of relation of economic, sociologic or demographics indicators or analysis of factors influencing dissemination of innovation, economic growth and many others. For the long time these analyses were predominantly done in the time domain. Empirical work mostly used and still uses regression and correlation methods [1, 2, 3], cointegration [4], codependence, causality monitoring, structural and vector models [5], and so on. Thus, examination of frequency characteristics was rather suppressed or marginal. In some cases the scientists in economy consider the dynamic behaviour as the motion of analysed inputs in terms of its leads and lags $[6,7]$. Another option is the frequency characteristics, such as dynamic correlation, which complement the knowledge from time domain in a suitable way and specify and validate the results from the time domain analysis [8-11].

Interconnection of analyses in both domains provides time-frequency (TF) techniques. Commonly used TF methods are Fourier Transform (FT), modifications of periodogram [12], TF varying autoregressive process (TFAR) [13], and continuous wavelet transform (CWT) $[14,15]$. The last one becomes very popular, especially in the last decade, for its good time resolution. We can examine in TF domain not only transform of inputs, but also co-movement of inputs. It can be done via instruments such as cross spectrum (WCS), co-spectrum or cohesion. These approaches propose more precise evaluation of co-movement to capture temporal character of co-movement [16-18] then simple time domain techniques.

If we focus on exploring economic phenomena, CWT is at the forefront of economic interest [19, 20]. Application of CWT helps with modelling and exploration of the joint movement of economic indicators. The advantages of CWT can be summarized in the following points: i) applicability on stationary and non-stationary time series; ii) possibility of time and frequency analysis in one, i.e. spectrogram or cospectrum analysis; iii) flexible choice of mother wavelet with respect to character of the inputs, iv) time resolution is adjusted to the scale, v) compared to other $\mathrm{TF}$ techniques, there is no need to optimize the parameters and thus limiting the quality of the resulting TF estimate. There is only discussion about wavelet and scale selection. As the disadvantage we can mention a more complex way of testing and identifying significant areas which is used for interpretation of the results. 
Together with implementation $\mathrm{TF}$ method into various disciplines arises the necessity of validation of the results. The basic works, giving instrument for significance testing, gives Torrence and Compo [21] followed by $\mathrm{Ge}$ [22]. Both works propose algorithm for testing wavelet power spectrum, cross-spectrum and linear coherence. Ge $[22,23]$ proved that the sampling period factor should be incorporated into testing to achieve numerically stable results. In all cases, authors' uses testing statistic with devoted distribution corrected with respect to the background spectrum distribution and sampling period. An alternative approach, presented in [15] or [14], uses Monte Carlo (MC) simulation for identification of critical values.

Based on the real data, we investigate the behaviour of testing procedure for the estimated wavelet cross spectrum with respect to the Gaussian white noise (GWN) background. We use both the statistical significance testing according $\mathrm{Ge}$ [22], and the identification of critical values using MC simulations. We deal with the advantages and disadvantages and provide recommendations for usage of TF co-movement significance testing. We follow up the paper [17] which works with significance testing of wavelet coefficients.

The paper is organized as follows: after the introduction, in the section Methodology, we introduce the theoretical background for co-movement testing. In the next section, Application, we describe data and present and summarize the achieved results. The paper ends with the Conclusion.

\section{Methodology}

In this section we present theoretical background for testing cross spectrum significance. We follow work of Torrence and Compo [21] and Ge [22]. Firstly, we present definition of wavelet cross spectrum (WCS). Consequently, we describe algorithm for statistical testing according to $\mathrm{Ge}$ [22] and according to $\mathrm{MC}$ simulations.

\subsection{Wavelet cross spectrum (WCS)}

An analysis of two time series co-movement can be done via WCS. The WCS between two time series/signals can be define as

$$
C_{\mathrm{xy}}(\mathrm{a}, b)=W_{x}^{*}(\mathrm{a}, b) W_{y}(\mathrm{a}, b),
$$

where $W_{x}(\mathrm{a}, b)$ and $W_{y}(\mathrm{a}, b)$ are the coefficients of wavelet transform corresponding to the time series $x(t)$ and $y(t)$ which are both independent GWN with corresponding variances $\sigma_{\mathrm{x}}{ }^{2}$ and $\sigma_{\mathrm{y}}{ }^{2}$. The symbol $*$ denotes complex conjugation, $a$ and $b$ are scale and time variables [22]. Since the WCS is complex, such in our case, when the complex Morlet wavelet are used, we can define its square absolut value, i.e. wavelet power cross spectrum, as

$$
\left|C_{x y}(\mathrm{a}, b)\right|^{2}=\left|W_{x}(\mathrm{a}, b)\right|^{2}\left|W_{y}(\mathrm{a}, b)\right|^{2},
$$

where $\left|W_{x}(\mathrm{a}, b)\right|^{2}$ and $\left|W_{y}(\mathrm{a}, b)\right|^{2}$ are the squared absolut value of WCS, i.e. wavelet power spectras [21].

\subsection{Significance test}

Let us have two time series $x(t)$ and $y(t)$, which are both independent GWN with corresponding variances $\sigma_{\mathrm{x}}{ }^{2}$ and $\sigma_{\mathrm{y}}{ }^{2}$, and wavelet spectra $W_{x}(\mathrm{a}, b)$ and $W_{y}(\mathrm{a}, b)$. As presented in [21], the square of the absolut value of normally distributed variable is $\chi^{2}(v)$ distribution with $v=2$ degrees of freedom. As presented in [22], Wells et al. [24] obtained the probability distribution of two independent non-central $\chi^{2}$-distributed random variable. In the special case, when both wavelet power spectras are $\chi^{2}(2)$ and non-centrality parameters are both zero, i.e. when

$$
\begin{aligned}
& \left|W_{x}(\mathrm{a}, b)\right|^{2} /\left(\delta \mathrm{t} \sigma_{\mathrm{x}}{ }^{2} / 2\right) \sim \chi^{2}(2), \\
& \left|W_{y}(\mathrm{a}, b)\right|^{2} /\left(\delta \mathrm{t} \sigma_{\mathrm{y}}{ }^{2} / 2\right) \sim \chi^{2}(2),
\end{aligned}
$$

where $\delta t$ is the sampling period, for their product holds

$$
\left|C_{x y}(\mathrm{a}, b)\right|^{2} /\left(\sigma_{\mathrm{x}}^{2} \sigma_{\mathrm{y}}^{2}\right) \sim 0.25 \delta t^{2} W_{2},
$$

where, based on Wells et al. [24], $W_{2}$ denotes probability distribution with probability density function

$$
f(z)=0.5 \mathrm{~K}_{0}\left(z^{1 / 2}\right) .
$$

Here $z$ is the random variable, and $K_{0}(z)$ is the modified Bessel function of order zero when the complex wavelets are used $[22,24]$.

\subsection{Monte Carlo (MC) simulations}

The idea of MC simulations is based on repeated random sampling to achieve numerical results. It uses statistics of original data for the subsequent replication of original data by generating large number of random realization of a stochastic process. On the basis of law of large numbers we can identify empirical distribution close to probabilistic distribution and identify empirical statistics, such as quantile or percentile. Resulting simulated process is consequently approximated by averaging these sub realizations in some way. We are going to apply MC simulations consisting from following steps [25]:

- Step 1: Definition of the model to be simulated

- Step 2: Generating random values of predefine distribution

- Step 3: Calculation with large number of repetitions

- Step 4: Analysis of results, quantiles identification

\section{Application}

For the demonstration we selected real economic data rather than their simulation, because such real data contain structural breaks and reactions on economic even. In case of its simulation some of unpredictable factor can be supressed, which is not welcomed.

Our approach consists from several steps. In the first step we estimate WCS and wavelet power cross 
spectrum (eq. (2)). In the second step we identify significant regions according to $\mathrm{Ge}$ [22] approach (eq. (5)). In the third step we used 1.000 Monte Carlo (MC) simulation to identify empirical critical values. At the end we compare both results (according to $\mathrm{Ge}$ and $\mathrm{MC}$ simulation) and formulate recommandations.

\subsection{Data}

For the demonstration of discussed approach we use seasonally adjusted quarterly data of gross domestic product (GDP), volume index in OECD reference year 2010 (OECD [26]) of the United Kingdom (UK) in 1956/01-2016/03, Korea in 1970/Q2-2016/03 and Group of 7 (G7) in 1961/02-2016/03. All variables are in the first differences of the logarithms (Fig. 1a-c). The motivation for data selection were: i) sufficient data range (it is desirable to have detailed time resolution); ii) in case of UK and G7 we expect co-movement, because UK is a member of G7 and thus support validation of purposed method; iii) in case of Korea we expect lower level of co-movement with G7, because Korea is not the member of G7. Additionally, with respect to the current situation in Great Britain (tendency to withdrawal from the EU) we were interested into analysis before such even having character of structural break which will affect the data.

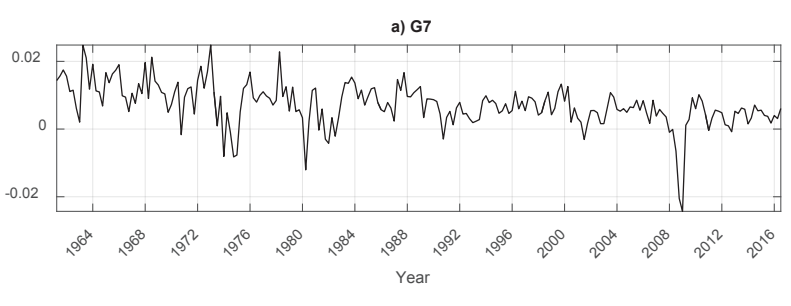

b) UK

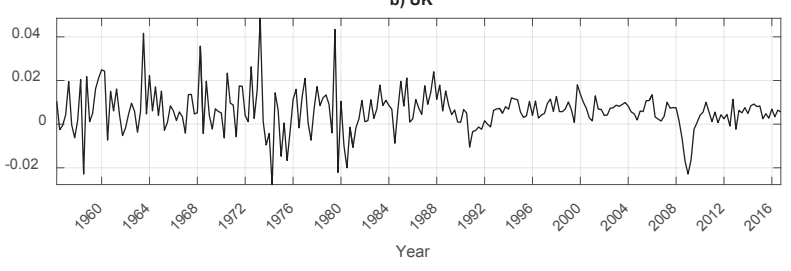

c) Korea

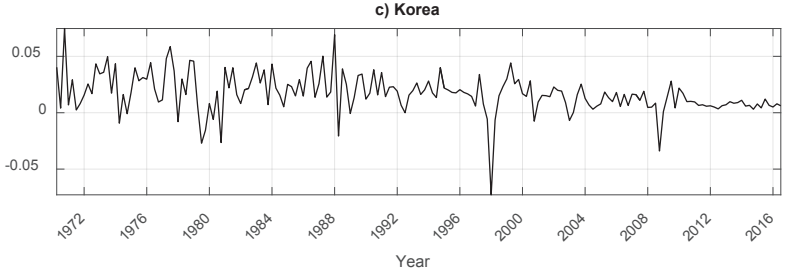

Fig. 1. Time series representation (1a: G7, 1b: UK, 1c: Korea)

\subsection{Setting of TF methods and MC}

For CWT estimation we used complex Morlet wavelet with central wavelet frequency $\mathrm{f}_{0}=1.5$ as the mother wavelet. We set scales corresponding to the range from 2 years to 40 years cycles, with 334 individual scales. The power WCS were calculated according to the formula (2). The resulting figures for the data can be seen in Fig. 2a-c.
In case of $\mathrm{MC}$ simulations we firstly generate two independent GWN series. Then, we estimate its CWTs and consequently power WCSs with 334 individual scales (corresponding to the data setting). Consequently, we generate 1.000 repetitions with the length of 1.000 points. Not to complicate things for the CWT with cone of influence we selected a time point in middle of WCS spectrogram. For each frequency (scale) from all MC iterations we save the obtained values. After that, we calculated $95 \%$ quantile of empirical distribution separately for each vector (scale).

\subsection{Results}

The results of co-movements are presented in Fig. 2a-c below. All figures have $x$-axis: the time, $y$-axis: the frequency measured in the cycles per year and $z$-axis: the power WCS. The $y$-axis was re-calculated from frequency to the cycles per year for the better interpretation of an economic inputs. Thus, given the quarterly character of the data, we denote the sampling frequency $F_{s}$ to be 4 samples per year. The red curve in the figures defines significant area identify via the MC simulations, the yellow dashed curve defines significant area according to Ge [22].

Focusing on the results of co-movement (Fig. 2a-c) with respect to the identification of significant area via Ge [22] we can state following. All figures show existence of co-movement between countries which matches our assumptions. That is, the UK-G7 (Fig. 2a) has higher level of co-movement caused by being UK in G7. The Korea and G7 (Fig. 2b) has less areas with comovement, but generally both UK-G7 and Korea-G7 has co-movement in the time of oil crisis 1970-1982 and in the time of financial crisis 2001-2010. Such strong comovement of Korea with G7 is also visible in Fig. 2c. All these significant co-movement (dashed curves in the Fig. 2a-c) were identified for long cycles, i.e. 10 years length cycles and longer. These results were confirmed by MC simulations.

Focusing on same figures (Fig. 2a-c), but on significant areas identified by the MC simulation, we can see an additional areas in all measured co-movements. That is, the additional co-movement between UK-G7 (Fig. 2a) in the time of oil crisis 1968-1982, but for shorter cycles, i.e. for the cycles of the approximately 24 years. Such additional co-moved area reflects the faster response in the country economy to the oil crisis, such as small and medium-sized enterprises, loans, investments etc. In the case of the co-movement between Korea-G7 (Fig. 2b) we can see also additional co-movement in the period 1974-1982 but for cycles of the length 4-6 years. Similarly, between Korea-UK (Fig. 2c) there is also the additional co-movement in the period 1972-1980 for cycles of the length 4-6 years. Here, the results again confirm our expectation of direct interconnection of UK and G7 and the weaker interconnection of Korea and G7. In all the cases results confirm the globalisation of the economies and the stronger impact of the oil crisis on the world economy then the financial crisis. 

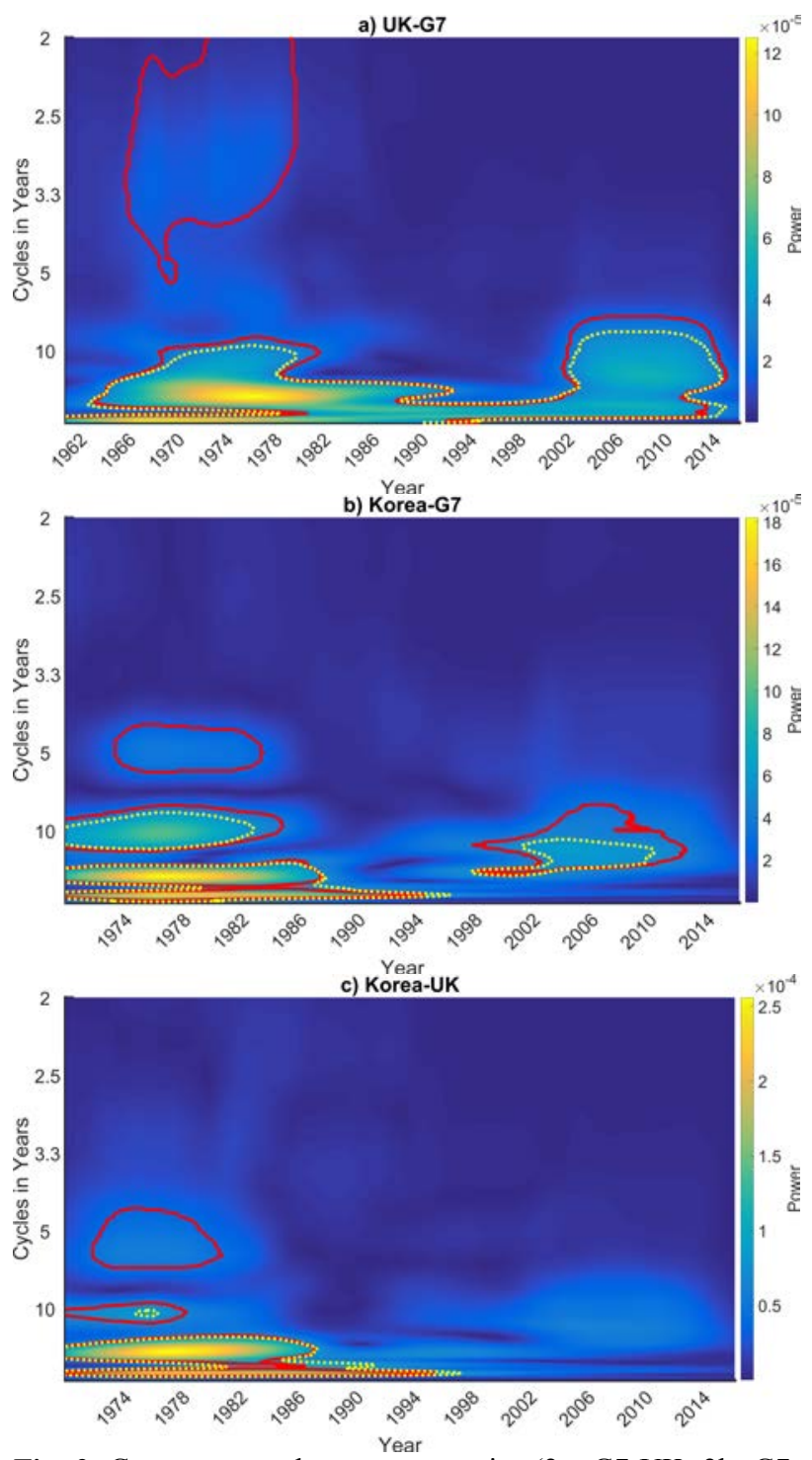

Fig. 2. Co-movement between countries (2a: G7-UK, 2b: G7Korea, 2c: Korea-UK)

The comparison and the verification of the accuracy and suitability of Ge approach via the MC simulations is presented on Fig. 4. For the illustration of the significance level we firstly preselect the three time slices of the WCS (for example of UK-G7 (Fig. 3)). Selected time slices are denoted by the numbers 1-3 and corresponds to the time 1976, 1998 and 2006. The figure (Fig. 4) demonstrates power WCS curves and the significance level identified by Ge (dashed-dotted line) and by the MC simulations (stars). We can see that the MC significance level is not a constant, resp. it is decreasing with the frequency. Thus, the MC simulation enables the additional power WCS components to be significant.

The difference between significant level of Ge and the MC simulations is most likely caused by the heteroscedastic character of an input data, when in the time period of commoved series are the sub-periods with the different variances (you can see Fig. 1). An additional source of the difference between the significance level could be the scale range selection.
Therefore, it is suitable to evaluate and assess obtained results in the context of application area (economy etc.).

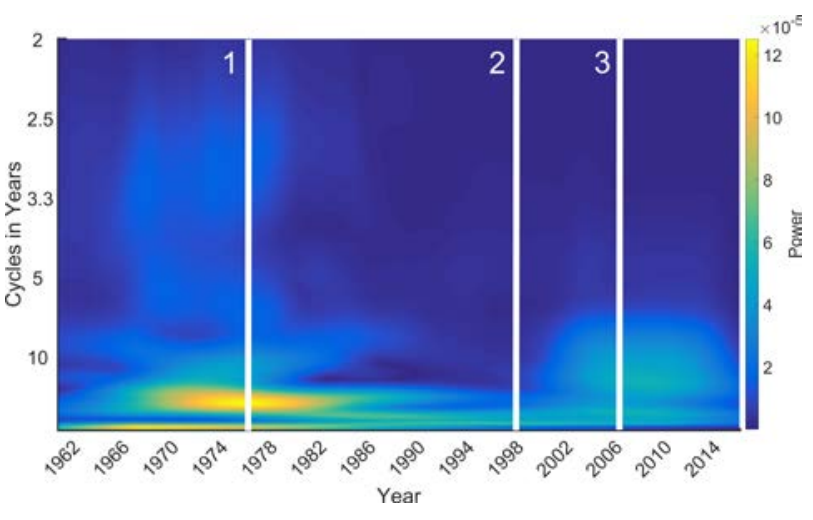

Fig. 3. Selection of slices in co-movement in three different time moments (1: 1976, 2: 1998, 3: 2006)

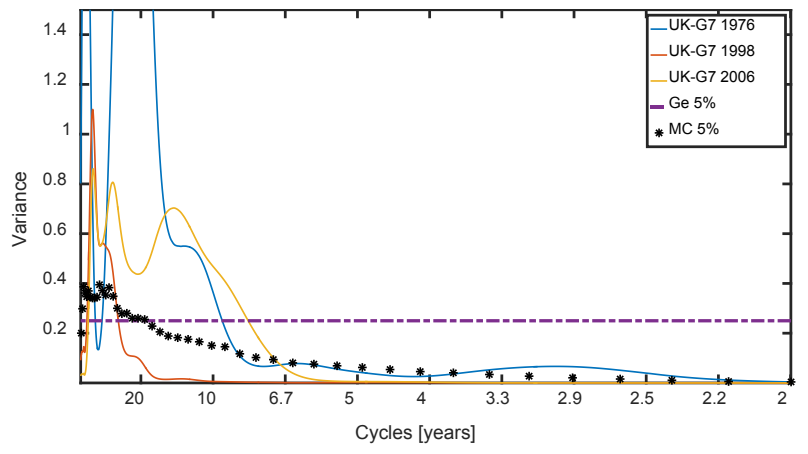

Fig. 4. Comparison of Ge and MC significance level

\section{Conclusion}

Using real data we analysed the behaviour of testing approach for the estimated wavelet power cross spectrum with respect to the GWN background. We use two approaches, namely the statistical significance according $\mathrm{Ge}$, and the identification of critical value using MC simulations. While Ge uses comparison with constant critical value identified via the modified Bessel function of order zero corresponding to the risk, the MC simulation identify critical value on the basis of repeated random sampling to identify empirical distribution.

On the basis of obtained results we suggest using MC simulations to identify critical values for cross spectrum rather than Ge approach especially when the data are heteroscedastic. It means in the case, when the data have the different variance during its time evolution. In such case, the Ge approach cannot be able to capture all significant commoved areas. As supporting step we see as suitable to assess obtained results in the context of the scientific area from which the data originate.

The research described in the paper was supported by the Czech Science Foundation via grant n. 17-24309S and by the Czech Ministry of Education in the frame of National 
Sustainability Program under grant LO1401. For research, the infrastructure of the SIX Center was used.

\section{References}

1. I. Korhonen, A. Peresetsky, What influences stock market behavior in Russia and other emerging countries, Emergin Market Finance \& Trade, 52 (2016)

2. J. Janku, S. Kappel, Z. Kucerova. Monetary and Fiscal Policy Coordination in Slovakia: A Game Theory Approach, Ekonomický časopis 63 (2015)

3. S. Kapounek, Z. Kučerová, J. Fidrmuc., Lending conditions in EU: The role of credit demand and supply, Economic Modelling (2017)

4. G. Hassan, A. Cooray, M. Holmes, The effect of female and male health on economic growth: crosscountry evidence within a production function Framework, Empirical Economics, 52 (2017)

5. M. Feldkircher, I. Korhonen, The rise of China and james charles its implications for the global economy:evidence from a global vector autopregressive model. Pacific Economic Review, 19 (2014)

6. E. Lukmanova, G. Tondl, Macroeconomic imbalances and business cycle synchronization. Why common economic governance is imperative for the Eurozone. Economic Modelling 62 (2017

7. F. Lu, H. Qiao, S. Wang, K. K. Lai, Y. Li, Timevarying coefficient vector autoregressions model based on dynamic correlation with an application to crude oil and stock markets. Environmental Research 152 (2017)

8. Ch. Croux, M. Forni, L. Reichlin, A Measure of Comovement for Economic Variables: Theory And Empirics. The Review of Economics and Statistics 83 (2001)

9. A. Iacobucci, A. Noullez, A frequency selective filter for short-length time series. Computational Economics 25 (2005)

10. J. Fidrmuc, T. Ikeda, K. Iwatsubo, International transmission of business cycles: Evidence from dynamic correlations. Economic Letters 114 (2012)

11. J. Poměnková, S. Kapounek, R. Maršálek, Variability of Dynamic Correlation-The Evidence of Sector-Specific Shocks in V4 Countries. Prague Economic Papers 23 (2014)

12. A. Xu, S. Haykin, R. J. Racine, Multiple Window Time-Frequency Distribution and Coherence of EEG Using Slepian Sequences and Hermite Function. IEEE Transactions of Biomedical Engineering 46 (1999)

13. J. G. Proakis, Ch. M. Rader, F. L. Ling, Ch. L. Nikias, M. Moonen, J. K. Proudler. 2002. Algorithms for Statistical Signal Processing. (Prentice Hall. 2002).

14. D. Wang, S. Shilong, W. Tse Peter., A general sequential Monte Carlo method based optimal wavelet filter: A Bayesian approach for extracting bearing fault features. Mechanical Systems and Signal Processing 52 (2015)
15. W. Jiang, S. Mahadevan, Wavelet spectrum analysis approach to model validation of dynamic systems. Mechanical Systems and Signal Processing 25 (2011)

16. R. Maršálek, J. Poměnková, S. Kapounek. A Wavelet-Based Approach to Filter Out Symmetric Macroeconomic Shocks. Comput. Econ. 44 (2014)

17. E. Klejmová, T. Malach, J. Poměnková, Wavelet Significance Testing with Respect to GWN Background: Monte Carlo Simulation Usage. In Proceedings of 27th international conference Radioelektronika 2017.

18. E. Klejmová, J. Poměnková, J. Blumenstein, Combination of the time-frequency representation for background noise supression. In Proceedings 31st European Conference on Moelling and Simulation ECMS 2017.

19. Z. Ftiti, A. Tiwari, A. Belanés, Tests of Financial Market Contagion: Evolutionary Cospectral Analysis V. S. Wavelet Analysis. Computational Economics 46 (2014)

20. A. N. Berdiev, Ch-P. Chang, Business cycle synchronization in Asia-Pacific: New evidence from wavelet analysis. Journal of Asia Econ. 37 (2015)

21. Ch. Torrence, G. P. Compo, A practical guide to wavelet analysis. Bulletin of the American Meteorological society 79 (1998)

22. Z. Ge, Significance tests for the wavelet cross spectrum and wavelet linear coherence. Annales Geophysicae 26 (2008)

23. Z. Ge, Significance tests for the wavelet power and the wavelet power spectrum. Annales Geophysicae 25 (2007)

24. W. T. Wells, R. L. Anderson, J. W. Cell, The distribution of the productof two central or noncentral chi-square varietes, Ann. Math. Stat. 33 (1962)

25. C. P. Robert, G. Casella, Monte Carlo Statistical Methods (New York: Springer, 2004)

26. Organisation for Economic Co-operation and Development: National Accounts [online database]. (2017) [cit. 2017-01-12]. Available at: http:\$backslash $\backslash$ backslash\$stats.oecd.org\$ $\$ \backslash\$Index.aspx?DatasetCode $\$=\$ S N A \backslash$ TAB LE1. 